\title{
n.03 2020
}

\section{Working Paper \\ of Public Health}

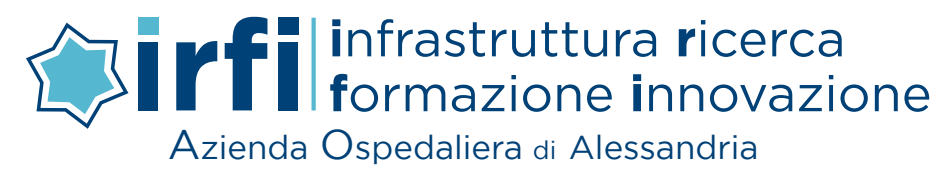


La serie di Working Paper of Public Health (WP) dell'Azienda Ospedaliera di Alessandria è una serie di pubblicazioni online ed Open Access, progressiva e multi disciplinare in Public Health (ISSN: 2279-9761). Vi rientrano pertanto sia contributi di medicina ed epidemiologia, sia contributi di economia sanitaria e management, etica e diritto. Rientra nella politica aziendale tutto quello che può proteggere e migliorare la salute della comunità attraverso l'educazione e la promozione di stili di vita, così come la prevenzione di malattie ed infezioni, nonché il miglioramento dell'assistenza (sia medica sia infermieristica) e della cura del paziente. Si prefigge quindi l'obiettivo scientifico di migliorare lo stato di salute degli individui e/o pazienti, sia attraverso la prevenzione di quanto potrebbe condizionarla sia mediante l'assistenza medica e/o infermieristica finalizzata al ripristino della stessa.

Gli articoli pubblicati impegnano esclusivamente gli autori, le opinioni espresse non implicano alcuna responsabilità da parte dell'Azienda Ospedaliera "SS. Antonio e Biagio e Cesare Arrigo" di Alessandria.

La pubblicazione è presente in: Directory of Open Access Journals (DOAJ); Google Scholar; Academic Journals Database:

\section{Comitato Scientifico:}

Prof. Roberto Barbato

Dott.ssa Manuela Ceccarelli

Dott. Diego Gazzolo

Dott.ssa Federica Grosso

Prof. Marco Krengli

Prof.ssa Roberta Lombardi

Prof. Leonardo Marchese

Prof. Vito Rubino

Dott. Gioel Gabrio Secco

Dott. Paolo Tofanini
Dott. Giacomo Centini

Dott. Gianfranco Ghiazza

Dott.ssa Daniela Kozel

Dott. Marco Ladetto

Dott. Antonio Maconi

Dott. Alessio Pini Prato

Dott.ssa Mara Scagni

Dott.ssa Maria Elena Terlizzi

Dott.ssa Roberta Volpini il WP (i.e. peer review). L'utilizzo del peer review costringerà gli autori ad adeguarsi ai migliori standard di qualità della loro disciplina, cosi come ai requisiti specifici del WP. Con questo approccio, si sottopone il lavoro o le idee di un autore allo scrutinio di uno o più esperti del medesimo settore. Ognuno di questi esperti fornirà una propria valutazione, includendo anche suggerimenti per l'eventuale miglioramento, all'autore, così come una raccomandazione esplicita al Comitato editoriale su cosa fare del manoscritto (i.e. accepted o rejected).

Al fine di rispettare criteri di scientificità nel lavoro proposto, la revisione sarà anonima, così come l'articolo revisionato (i.e. double blinded).

\section{Diritto di critica:}

Eventuali osservazioni e suggerimenti a quanto pubblicato, dopo opportuna valutazione di attinenza, sarà trasmessa agli autori e pubblicata on line in apposita sezione ad essa dedicata.

Questa iniziativa assume importanza nel confronto scientifico poiché stimola la dialettica e arricchisce il dibattito su temi d'interesse. Ciascun professionista avrà il diritto di sostenere, con argomentazioni, la validità delle proprie osservazioni rispetto ai lavori pubblicati sui Working Paper of Public Health.

Nel dettaglio, le norme a cui gli autori devono attenersi sono le seguenti:

- I manoscritti devono essere inviati alla Segreteria esclusivamente in formato elettronico all'indirizzo e-mail dedicato

- A discrezione degli autori, gli articoli possono essere in lingua italiana o inglese. Nel caso in cui il manoscritto è in lingua italiana, è possibile accompagnare il testo con due riassunti: uno in inglese ed uno in italiano, cosi come il titolo;

- Ogni articolo deve indicare, le Keywords, nonché il tipo di articolo (i.e. Original Articles, Brief Reports oppure Research Reviews);

Comitato editoriale:

Dott. Antonio Maconi

Dott. Alfredo Muni

Dott.ssa Marinella Bertolotti

\section{Responsabile:}

Dott. Antonio Maconi

telefono: +39.0131.206818

email: amaconi@ospedale.al.it

\section{Segreteria:}

Mariateresa Dacquino, Marta Betti,

Mariasilvia Como, Laura Gatti

telefono: +39.0131.206192

email: mdacquino@ospedale.al.it; Igatti@ospedale.al.it

\section{Norme editoriali:}

Le pubblicazioni potranno essere sia in lingua italiana sia in lingua inglese, a discrezione dell'autore. Sarà garantita la sottomissione di manoscritti a tutti coloro che desiderano pubblicare un proprio lavoro scientifico nella serie di WP dell'Azienda Ospedaliera di Alessandria, purché rientrino nelle linee guida editoriali. II Comitato editoriale verificherà che gli articoli sottomessi rispondano ai criteri editoriali richiesti. Nel caso in cui lo si ritenga necessario, lo stesso Comitato editoriale valuterà l'opportunità o meno di una revisione a studiosi o ad altri esperti, che potrebbero o meno aver già espresso la loro disponibilità ad essere revisori per
- L'abstract è il riassunto dell'articolo proposto, pertanto dovrà indicare chiaramente: Obiettivi; Metodologia;

- Gli articoli dovrebbero rispettare i seguenti formati: Original Articles (4000 parole max., abstract 180 parole max., 40 references max.); Brief Reports (2000 parole max., abstract 120 parole max., 20 references max., 2 tabelle o figure) oppure Research Reviews (3500-5000 parole, fino a 60 references e 6 tabelle e figure);

- I testi vanno inviati in formato Word (Times New Roman, 12, interlinea 1.5). Le note, che vanno battute in apice, non possono contenere esclusivamente riferimenti bibliografici. Inoltre, la numerazione deve essere progressiva;

- I riferimenti bibliografici vanno inseriti nel testo riportando il cognome dell'Autore e l'anno di pubblicazione (e.g. Calabresi, 1969). Nel caso di più Autori, indicare nel testo il cognome del primo aggiungendo et al; tutti gli altri Autori verranno citati nei riferimenti bibliografici alla fine del testo.

- I riferimenti bibliografici vanno elencati alla fine del testo in ordine alfabetico (e cronologico per più opere dello stesso Autore).

Nel sottomettere un manoscritto alla segreteria di redazione, l'autore accetta tutte le norme qui indicate.
- Risultati; Conclusioni; 


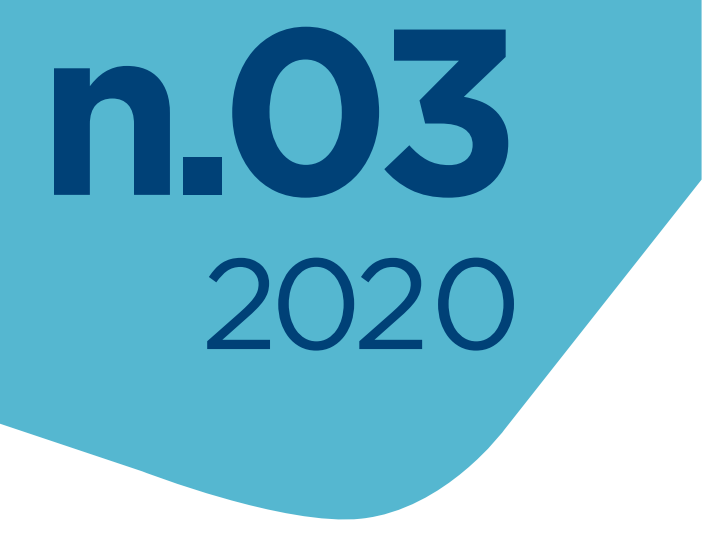

titolo

IL RUOLO DELLA TECNICA STEREOTASSICA NELLA MODERNA RADIOTERAPIA: PROTOCOLLO DELLA S.C. DI RADIOTERAPIA ASO ALESSANDRIA SS. ANTONIO E BIAGIO E CESARE ARRIGO

title

THE ROLE OF TECHNOLOGY IN THE MODERN STEREOTACTIC RADIOTHERAPY:

PROTOCOL OF THE S.C. OF RADIOTHERAPY ASO ALESSANDRIA SS. ANTONIO AND BIAGIO AND CESARE ARRIGO

autori
A. Pastorino, E. Garibaldi*, O. Durante*, A. Fozza*,
L. Berretta*, G. Pozzi*, E. Cazzulo**, M.P. Barbero**,
P. Franzone*.
*Azienda Ospedaliera SS. Antonio e Biagio e Cesare Arrigo,
S. C. Radioterapia - Alessandria
**Azienda Ospedaliera SS. Antonio e Biagio e Cesare Arrigo,
S.C Fisica Sanitaria - Alessandria

-Scuola di Specializzazione in Radioterapia, Università degli Studi di Genova

tipologia

Research Review

keywords

Radiation therapy, stereotactic, SBRT, SRS, SFRT, cancer 


\section{TITOLO:}

IL RUOLO DELLA TECNICA STEREOTASSICA NELLA MODERNA RADIOTERAPIA:

PROTOCOLLO DELLA S.C. DI RADIOTERAPIA ASO ALESSANDRIA SS. ANTONIO E BIAGIO E CESARE ARRIGO

\section{TITLE:}

THE ROLE OF TECHNOLOGY IN THE MODERN STEREOTACTIC RADIOTHERAPY:

PROTOCOL OF THE S.C. OF RADIOTHERAPY ASO ALESSANDRIA SS. ANTONIO AND BIAGIO AND CESARE ARRIGO

\section{AUTORI:}

A. Pastorino, E. Garibaldi*, O. Durante*, A. Fozza*, L. Berretta*, G. Pozzi*,

E. Cazzulo**, M.P. Barbero**, P. Franzone*.

*Azienda Ospedaliera SS. Antonio e Biagio e Cesare Arrigo, S.C.

Radioterapia - Alessandria

${ }^{* *}$ Azienda Ospedaliera SS. Antonio e Biagio e Cesare Arrigo, S.C. Fisica Medica - Alessandria

'Scuola di Specializzazione in Radioterapia - Università degli Studi di Genova

\section{TIPOLOGIA:}

Research Review

\section{KEYWORDS:}

Radiation therapy, stereotactic, SBRT, SRS, SFRT, cancer 


\section{ABSTRACT}

OBIETTIVI: L'obiettivo di questo elaborato è la stesura di un protocollo uniforme all'interno della nostra Struttura Complessa di Radioterapia per l'esecuzione del trattamento radiante di tipo stereotassico.

METODOLOGIA: Abbiamo raccolto le diverse esperienze personali dei componenti del Team di Radioterapia, unedole ad una revisione di letteratura di articoli riguardanti la tecnica stereotassica, tenendo conto dell indicazioni fornite dalla Società italiana di Radioterapia (AIRO).

RISULTATI: E' stato possibile delineare uno schema suddiviso per distretto corporeo atto a omogeneizzare ed uniformare il trattamento radiante stereotassico all'interno del nostro reparto.

CONCLUSIONI: Questo lavoro ci ha permesso di ottenere una migliore organizzazione del lavoro nella preparazione, esecuzione e rivalutazione post-trattamento riguardo ad una tecnica da poco introdotta nella Nostra Struttura Complessa.

\section{ABSTRACT}

AIMS: The aim of this paper is the drafting of a uniform protocol within our Complex Radiotherapy Structure for the execution of stereotaxic radiant treatment.

METHODS: We collected the different personal experiences of the members of the Radiotherapy Team, with a review of the literature of articles concerning the stereotaxic technique, taking into account the indications provided by the Italian Society of Radiotherapy (AIRO).

RESULTS: It was possible to outline a scheme divided by body district designed to homogenize and standardize the stereotactic radiant treatment within our department.

CONCLUSIONS: This work has allowed us to obtain a better organization of the work in the preparation, execution and post-treatment reassessment regarding a technique recently introduced in our Department. 


\section{PARTE GENERALE}

\section{INTRODUZIONE}

Il concetto di radioterapia stereotassica fu descritto per la prima volta da Lars Leksell nella metà del XX secolo, il quale aveva concepito un metodo per somministrare alte dosi in maniera precisa e focalizzata all'encefalo. Questa metodica prese il nome di radiochirurgia stereotassica (SRS).

Nei successivi 25 anni la tecnica fu progressivamente affinata sino ad arrivare alla "gamma Knife" messa a punto dal gruppo del Karolinska Hospital di Stoccolma. Con questa tecnica era possibile il trattamento delle localizzazioni metastatiche con la somministrazione di dosi ablative in una singola seduta, mediante l'utilizzo di un "caschetto stereotassico" fissato al cranio per la localizzazione delle coordinate stereotassiche.

La filosofia di un trattamento che prevedesse la somministrazione di alte dosi al tumore con massimo risparmio degli organi critici circostanti, grazie ai più moderni sistemi di immobilizzazione, di verifica (IGRT) e di treatment planning (IMRT) si è poi evoluto in proposte di trattamenti stereotassici frazionati, che comunque consentissero una precisa riproducibilità giornaliera del trattamento e l'utilizzo di una tecnica radioterapica altamente conformata (SFRT).

\subsection{Applicazioni cliniche della Radioterapia Stereotassica Cranica (SRS/SFRT)}

Le Metastasi cerebrali sono una comune complicazione neurologica di una malattia neoplastica sistemica e sono causa di importanti morbidità e mortalità.

Le metastasi encefaliche rappresentano la localizzazione tumorale intracranica più frequente: I'incidenza di diagnosi di metastasi cerebrali è 3-10 volte più frequente dell'incidenza di diagnosi di nuovi tumori primitivi cerebrali. L'incidenza è aumentata nel tempo come risultato del miglioramento della diagnostica neuroradiologica e dei trattamenti sistemici. La maggior parte dei pazienti che sviluppano secondarismi cerebrali hanno una ridotta aspettativa di vita, ma pazienti con malattia intracranica limitata possono avere outcomes più favorevoli con terapie locali intensive, quali la $\mathbf{N C H}$ e la SRS/SFRT. 
Le neoplasie che più frequentemente metastatizzano sono quelle della mammella, del polmone e i melanomi.

Mammella: circa il $15-25 \%$ delle pazienti affette da tumore mammario sviluppa metastasi cerebrali. Fattori di rischio sono: giovane età, stato recettoriale negativo, malattia poco differenziata, alto grading ed HER $2+$.

I pazienti affetti da tumore polmonare non a piccole cellule sviluppano metastasi cerebrali nel $22-54 \%$ dei casi. L'incidenza è più elevata nei casi con mutazioni EGFR, che tuttavia si associa a maggiore sopravvivenza.

In circa il $10 \%$ dei pazienti con microcitoma polmonare le metastasi sono presenti già alla diagnosi e un altro $40-50 \%$ le svilupperà successivamente.

Nei pazienti con melanoma avanzato si possono verificare metastasi cerebrali nel $40-50 \%$ dei casi.

\section{- SRS/SFRT VS NCH:}

Molti studi comparano la SRS con la NCH riportando simili outcomes, tuttavia in presenza di bias; non sono a oggi disponibili studi randomizzati. La SRS è considerata meno invasiva, può essere somministrata ambulatorialmente e ha un miglior rapporto costi-benefici rispetto alla $\mathrm{NCH}$. I pazienti con lesioni di maggiori dimensioni possono necessitare della somministrazione cronica di steroidi.

\section{- WBRT dOPO (NCH o) SRS/SFRT:}

II razionale della WBRT dopo NCH O SRS è sterilizzare eventuali foci microscopici intracranici di malattia, in sede di intervento e/o a distanza, in pazienti con un limitato numero di lesioni. II ruolo di questo trattamento è stato molto dibattuto.

Tre studi di fase III e una meta-analisi hanno dimostrato che l'omissione della WBRT risulta in un peggiore controllo di malattia intracranica locale e a distanza, tuttavia non ha effetti sulla sopravvivenza. Una recente meta-analisi di 3 studi randomizzati che hanno confrontato la SRS da sola con la SRS + WBRT in pz con 1-4 lesioni ha suggerito un vantaggio in sopravvivenza per la SRS da sola nei pazienti con meno di 50 anni, senza diminuzione del rischio di nuove metastasi con l'aggiunta delle WBRT. Al 
contrario nei pazienti con età maggiore di 50 anni la WBRT è risultata diminuire il rischio di nuove metastasi ma non la sopravvivenza.

La WBRT adiuvante alla $\mathrm{NCH}$ riduce il rischio di ricaduta intracranica locale e a distanza nei pz con metastasi maggiori di $3 \mathrm{~cm}$ e/o malattia sistemica attiva.

Uno studio randomizzato di fase III ha confrontato la SRS da sola vs la SRS+WBRT in pz con 1-3 lesioni encefaliche analizzando come end-point primario la funzione neurocognitiva (peggioramento rispetto al baseline in 6 test eseguiti nei tre mesi dopo la terapia). II declino è stato significativamente più frequente dopo la SRS+WBRT rispetto alla SRS da sola (88\% vs 62\%). L'analisi della QoL del trial EORTC 22952-26001 ha dimostrato ad 1 anno di FU non significative differenze nello stato globale di salute dei pazienti ma ha riportato che i pz sottoposti a SRS+WBRT avevano uno stato cognitivo transitoriamente inferiore e maggiore fatigue.

Sulla base dei risultati di questo trial I'ASTRO ha raccomandato di non utilizzare routinariamente la WBRT adiuvante in pz con limitato numero di metastasi cerebrali (Choose Wisely-ASTRO 2014)

- SRS/SFRT pOSt NCH:

La SRS dopo NCH ha l'intento di diminuire le ricadute locali evitando le sequele cognitive della WBRT. Molti studi retrospettivi ed un prospettico di fase II hanno riportato un LC ad 1 anno di circa $80 \%$ ed una OS mediana di 10-17 mesi, suggerendo che la SRS post NCH è efficace come la WBRT nell'ottenere un LC. Un approccio alternativo è la SFRT, associata ad un più basso rischio di radionecrosi nelle lesioni di maggiori dimensioni.

Il bilancio tra rischi e benefici è attualmente sconosciuto ed esistono problematiche irrisolte quali: la dose ottimale, il frazionamento, gli effetti sulla OS, la QoL, e gli effetti sulle funzioni cognitive. Sono in corso studi randomizzati. II rischio di radionecrosi dopo SRS adiuvante alla $\mathrm{NCH}$ sembra essere più alto (9\%-17.5\%) che con la WBRT adiuvante alla NCH o SRS (2.6\%) e può aumentare negli anni (7\% ad 1 aa, $16 \%$ a 2 anni). C'è carenza di informazioni circa le manifestazioni cliniche conseguenti la radionecrosi (cefalea, crisi comiziali, emorragie). Uno dei rischi maggiori è la dipendenza cronica dallo steroide per controllare l'edema. La SRS 
post $\mathrm{NCH}$ è inoltre associata ad un maggior rischio di ricaduta leptomeningea (8-13\%), soprattutto nell' istologia mammaria e non si sa se la WBRT può ridurre tale rischio. In conclusione non esiste un elevato livello di evidenza per la SRS/SFRT post metastasectomia.

\subsection{Applicazioni cliniche della Radioterapia Stereotassica Body (SBRT)}

Sulla base dell'esperienza acquisita con il trattamento delle lesioni cerebrali a metà degli anni 90 venne messa a punto una tecnica di radioterapia stereotassica per la cura delle lesioni extracraniche (SBRT) con l'ausilio di presidi di immobilizzazione dedicati per il trattamento delle lesioni polmonari, epatiche e dello spazio retroperitoneale (bodyframe-system), siano esse primitive o metastatiche.

Venivano altresi utilizzati presidi quali i compressori addominali per minimizzare i movimenti d'organo dovuti alle escursioni respiratorie.

Oggi la SBRT è utilizzata per somministrare una radioterapia cosiddetta ipofrazionata in cui piccoli volumi vengono trattati ad alte dosi, tipicamente in 1-5 sedute totali, con l'utilizzo di acceleratori lineari dotati di collimatori multilamellari ed in grado di somministrare la dose con tecniche IMRT e IGRT.

La somministrazione della dose in singola seduta ha l'indubbio vantaggio di impattare in maniera meno significativa sulla lista d'attesa, di azzerare le problematiche di riproducibilità giornaliera del trattamento, specialmente se indaginosa e protratta nel tempo e quindi impegnativa per il paziente, e di consentire da un punto di vista radiobiologico una maggior probabilità di controllo del tumore agendo sul danno endoteliale della cellula tumorale che è indipendente dalla radiosensibilità del tumore. Ciò è di primaria importanza nel trattamento ad esempio delle localizzazioni metastatiche da tumori come il melanoma classicamente considerate radioresistenti.

\section{PAZIENTI CANDIDABILI AL TRATTAMENTO CON STEREOTASSI}

I pazienti candidabili al trattamento con SRS/SFRT/SBRT devono avere un buon Performance Status (ECOG 0) compatibile con le procedure di immobilizzazione che possono essere protratte nel tempo. 
In particolare il trattamento con SBRT delle lesioni metastatiche è proponibile in pazienti con presenza concomitante di altre lesioni, purché si tratti di un "setting" di pazienti cosiddetti oligometastatici/oligoricorrenti, con un controllo sistemico di malattia, aspettativa di vita a medio termine (> 3 mesi) e condizioni generali buone, come indicato dalla letteratura.

Da un punto di vista tecnico, l'AAPM TG 101, suggerisce di non proporre la SBRT come opzione di trattamento nel caso in cui ci siano nelle immagini TC degli artefatti metallici o di movimento che non permettano la localizzazione del target e degli organi a rischio con sufficiente accuratezza.

Possono quindi essere avviati a radioterapia stereotassica pazienti con lesioni metastatiche polmonari, epatiche, renali/surrenaliche, retroperitoneali, linfonodali, ossee, nonché pazienti con lesioni primitive polmonari, addominali non operabili.

Al momento però non includiamo nel nostro protocollo le lesioni epatiche e pancreatiche.

\section{SET UP E TC DI SIMULAZIONE}

L'unità di Radioterapia è dotata di presidi di immobilizzazione dedicati alla RT stereotassica che verranno scelti in base ai distretti anatomici da irradiare.

a) Cuscino di contenimento total body a vuoto agganciato ad apposite barre graduate in fibra di carbonio per irradiazione di lesioni toraciche, addominali o pelviche con possibilità di utilizzare anche compressore addominale (a fascia o a compressione diretta rigida). II compressore deve essere impiegato nel caso di lesioni toraciche e sottodiaframmatiche (addome superiore) per minimizzare l'escursione respiratoria. Nel caso di lesioni mobili, oltre al compressore, deve essere eseguita un TC 4D per poter delineare correttamente il volume da irradiare (ITV).

b) Sistema per IMRT testa collo (base in fibra di carbonio, appoggiatesta in plexiglass di diverse dimensioni e maschere testa/ testa spalle in materiale termoplastico rinforzato per testa-collo). Possibilità di cuscino modellabile a caldo per rendere più anatomico l'appoggiatesta. 
c) Sistema WING con appoggiatesta di diverse dimensioni per lesioni toraciche non polmonari.

d) Sistema PELVI con base in fibra di carbonio, supporto ginocchia e blocco delle caviglie in diverse inclinazioni. (da utilizzare per lesioni pelviche o per ritrattamenti in aree contigue a quelle già trattate mediante tale sistema di immobilizzazione).

e) Cuscini modellabili in poliuretano espanso rivestiti in poliestere di microfibra, di diverse dimensioni, agganciabili direttamente alle barre dei lettini (TC e LINAC) per lesioni di qualsiasi distretto corporeo compresi gli arti.

Le scansioni TC per la RT stereotassica devono essere di spessore $1 \mathrm{~mm}$ nella zona intorno al target, $3 \mathrm{~mm}$ lontano dal target.

L'estensione della TC deve essere tale da comprendere tutti gli organi critici del distretto irradiato, per una corretta valutazione del DVH.

Possibilità di eseguire contestualmente SIMULAZIONE VIRTUALE per ottenimento dell'isocentro definitivo.

Il dataset delle immagini TC del paziente verrà coregistrato con le altre immagini diagnostiche ritenute utili (RM, PET) da parte del fisico medico, con una fusione rigida o deformabile.

\section{CONTOURING E MARGINI}

Le incertezze riguardano: incertezza dovuto alla coregistrazioni delle immagini multimodali (MRI-CT, CT-CBCT): $\approx 1 \mathrm{~mm}$; incertezza posizionamento MLC: $0.5 \mathrm{~mm}$; incertezza isocentro: $0.5-1 \mathrm{~mm}$; incertezza lettino: 0.5-1 mm.

$\mathrm{Nel}$ caso della radioterapia stereotassica a seduta singola, l'incertezza maggiore è data dal contornamento del GTV e CTV. Per questo è fortemente consigliato utilizzare almeno due dataset di immagini multimodali, tutte eseguite a strato sottile.

C'è abbastanza accordo in letteratura di fissare il margine (ITV-CTVGTV)-PTV $\leq 3 \mathrm{~mm}$.

Delineare tutti gli altri OaR inclusi nella regione corporea. 


\section{PIANO DI CURA}

Approntati i piani di cura da parte del fisico, questi saranno discussi con il radioterapista e verrà scelto il piano di cura che sia un compromesso ottimale tra la complessità, la riproducibilità, il tempo di trattamento e l'omogeneità della dose somministrata nel massimo rispetto della dose ricevuta dagli organi critici.

\section{DOSE CONSTRAINTS}

Le tolleranze dei tessuti sani nel contesto dei regimi di dose ipofrazionata della radioterapia stereotassica si stanno continuamente evolvendo e a riguardo c'è un'esperienza limitata. In bibliografia, alla voce constraints di dose, le principali tabelle che seguiremo, oltre alle informazioni fornite dal QUANTEC.

\section{VERIFICA PRE-TRATTAMENTO}

Ogni piano di cura stereotassico deve essere testato alla macchina prima del trattamento stesso.

\section{EROGAZIONE}

Una volta approvato il piano di cura il paziente sarà pronto per la radioterapia all'unità di trattamento SYNERGY.

Il paziente sarà posizionato con il presidio di immobilizzazione scelto al momento della TC di simulazione e con il localizzatore Exapod.

Sarà selezionata la CLIP BOX che includerà il target volume e le strutture anatomiche adiacenti utili per l'esecuzione del matching, come indicato in ciascuna sezione della parte sistematica $\circ$ in alternativa come indicato dal medico radioterapista.

Verranno acquisite quindi immagini tramite CBCT del distretto sottoposto a irradiazione e opportunamente confrontate con quelle acquisite con la TC per piano di cura mediante il "matching" delle immagini stesse. II protocollo di fusione sarà indicato in ciascuna sezione della parte sistematica $\mathrm{o}$, in alternativa, sarà indicato dal medico radioterapista.

$\mathrm{Nel}$ caso di scostamento (entro $5 \mathrm{~mm}$ per le traslazioni ed entro $3^{\circ}$ per le rotazioni) il tecnico apporterà le necessarie correzioni del set-up e una 
volta che queste ultime siano state approvate dal radioterapista, il trattamento potrà aver luogo. In caso di scostamenti maggiori il paziente deve essere riposizionato o ripianificato per persistenza di scostamenti sopra il range stabilito.

\section{FOLLOW-UP}

I pazienti una volta congedati saranno sottoposti a visita di controllo a 30 giorni dal trattamento radioterapico al fine di valutare l'eventuale tossicità acuta.

In seguito, il primo esame di ristadiazione effettuato con la stessa metodica utilizzata prima del trattamento, in genere TC con mdc o RM, verrà effettuato a 60 giorni dal trattamento radioterapico per la valutazione della risposta ottenuta; in caso di esame PET esso sarà effettuato a 90 giorni dal trattamento.

I controlli clinici successivi avverranno a cadenza trimestrale e gli esami di ristadiazione organizzati in collaborazione con i curanti di riferimento.

\section{PARTE SISTEMATICA}

\section{OSSO}

\subsection{Metastasi Ossee Vertebrali:}

Criteri di Inclusione:

Lesioni coinvolgenti non più di un corpo vertebrale, distante $3-5 \mathrm{~mm}$ dal midollo, lesione ben evidente all'imaging, con conferma istologica del tumore primario.

\section{Criteri di Esclusione:}

Pazienti con lesioni a rischio di crollo vertebrale, prognosi $<3$ mesi, istologia di mieloma o linfoma, compressione midollare in atto 0 incipiente.

Imaging Pre-RT:

Al fine di consentire un accurato contouring del target volume e del midollo spinale deve essere eseguita TC di centratura in posizione di trattamento con slices 1,25 che sarà coregistrata con le immagini RM T1 
e T2 pesate dell'indagine diagnostica. In pazienti che non possano eseguire la RM (portatori di PM, pazienti con clips magnetiche, pazienti claustrofobici) dovrebbe essere eseguita una PET di centratura con radiofarmaco idoneo.

Per la delineazione del target volume si seguiranno le linee guida Internazionali [International Spine Radiosurgery Consortium (ISRC)]:

Si suddividono le vertebre nei seguenti settori: corpo vertebrale, peduncoli, apofisi trasversa, lamina vertebrale ed apofisi spinosa.

\| GTV corrisponde alla lesione visualizzata alla TC e alla RM.

Per la definizione del CTV (figura 1):

- l'intero corpo vertebrale deve essere irradiato nel caso di interessamento di ciascuna sua porzione o estensione extravertebrale;

- quando la neoplasia interessa il corpo vertebrale in posizione laterale,

il CTV deve comprendere anche il peduncolo e l'apofisi trasversa omolaterali. La presenza di interessamento diffuso dell'intero corpo vertebrale rappresenta indicazione all'irradiazione di entrambi i peduncoli e apofisi traverse;

- se la metastasi interessa il corpo vertebrale e un peduncolo, il CTV deve comprendere l'intero corpo vertebrale, il peduncolo, l'apofisi trasversa e la lamina omolaterali;

- nei casi in cui il corpo vertebrale ed entrambi i peduncoli/apofisi trasverse siano interessati dalla malattia, il CTV è rappresentato dall'intero corpo vertebrale e dai peduncoli, apofisi trasverse e lamine bilateralmente;

- quando la metastasi è localizzata solo a un peduncolo, il CTV deve comprendere, oltre a questo, anche l'apofisi trasversa e la lamina omolaterali \pm il corpo vertebrale;

- I'interessamento di una sola lamina fa sì che il CTV sia rappresentato, oltre che dalla lamina stessa, dal peduncolo/apofisi trasversa e spinosa omolaterali. Se il processo spinoso è interessato, il CTV deve includere, oltre a questo, entrambe le lamine.

Non è raccomandata l'estensione del CTV a livello epidurale in assenza di invasione meningea; non è consigliabile un volume circolare intorno al 
canale midollare in caso di interessamento del corpo vertebrale, dei peduncoli e dell'apofisi spinosa.

Figura 1: delineazione del CTV in base alla localizzazione della lesione

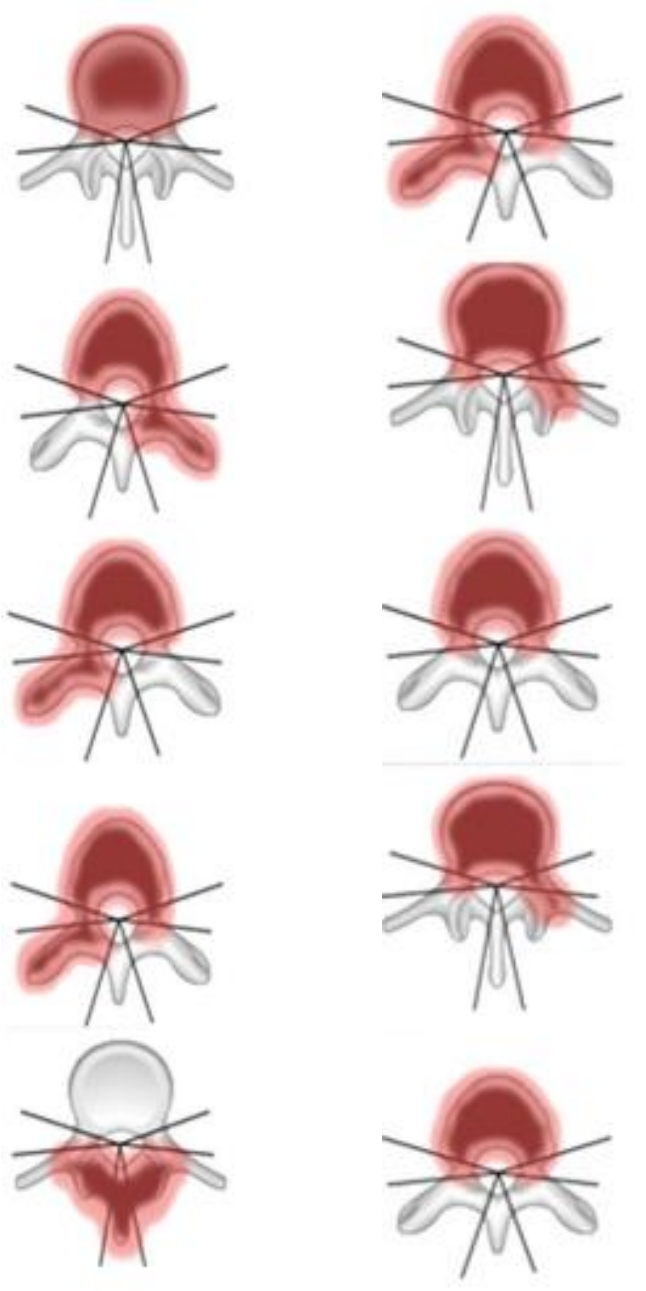

II PTV prevede l'aggiunta di un margine di 2-3 mm.

OAR: essenzialmente il midollo che va contornato con estensione di almeno una vertebra sopra e una sotto il PTV. Si aggiunge un margine di $3 \mathrm{~mm}$ per dare origine al PRV midollo.

Si delineano inoltre tutti gli OaR inclusi nella regione corporea. Dosi: 21-27 Gy in 3 frazioni, 30-35 Gy in 5 frazioni. 
Dose Constraints: $\mathrm{Ci}$ riferiamo ai constraints riportati dal documento AAPM TG 101, al position paper AIRO reperibile sul sito e alla consensus britannica sui constraints impiegati nei trattamenti stereotassici, che in parte adotta i limiti di dose riportati dal report AAPM 101 e in parte integra questi ultimi con dati derivanti da studi più recenti.

Nello specifico, per il midollo spinale 7 Gy, 12,3 Gy e 14,5 Gy in un volume inferiore a $1 \mathrm{~cm}^{3}$ per irradiazioni in 1,3 e 5 frazioni. Sono raccomandate anche dosi massime di $10 \mathrm{~Gy}, 18 \mathrm{~Gy}$ e $23 \mathrm{~Gy}$ in un volume inferiore a $0.35 \mathrm{~cm}^{3}$; è comunque tollerata una dose massima di 10 Gy al $10 \%$ del volume del midollo spinale.

Follow-Up: RMN a 2-3 mesi dalla SBRT.

\subsection{Metastasi Ossee Extravertebrali}

Criteri Di Inclusione: Lesioni in paziente oligometastico, ben evidenti all'imaging, conferma istologica del tumore primario.

Criteri Di Esclusione: Pazienti con lesioni a rischio di frattura, prognosi $<3$ mesi, istologia di mieloma o linfoma.

Imaging Pre-Rt: Per la corretta definizione della lesione, occorre effettuare una TC \pm RM, ed eventuale PET-TC (con idoneo radiofarmaco) GTV: malattia evidenziata dall'imaging (BTV/GTV).

CTV: aggiungere un margine di 3-5 mm PTV: aggiungere un margine di 2$3 \mathrm{~mm}$.

Delineare tutti gli altri OaR inclusi nella regione corporea.

Dosi: 21-27 Gy in $3 \mathrm{fx}, 30-35 \mathrm{fx}$ in $5 \mathrm{fx}$

Follow-Up: imaging utilizzato pretrattamento a 2-3 mesi dalla SBRT

\section{ENCEFALO}

\section{Selezione dei Pazienti}

Identificazione dei pazienti che realmente possano avere un beneficio da questo approccio.

DS-PGA (Diagnosis Specific-Graded Prognostic Assessment, Tabella 1) mette in relazione età, KPS, numero di metastasi, istologia, dimensioni della lesione. Gold standard per la selezione dei pz sono l'età $<60$ aa e KPS $<70$ con un buon controllo di malattia extracranica (Tabella 2). 
Tabella 1: schema di valutazione del beneficio in base alle caratteristiche del paziente e Mediana di sopravvivenza in base al calcolo del DS-GPA Score

\section{DS-GPA}

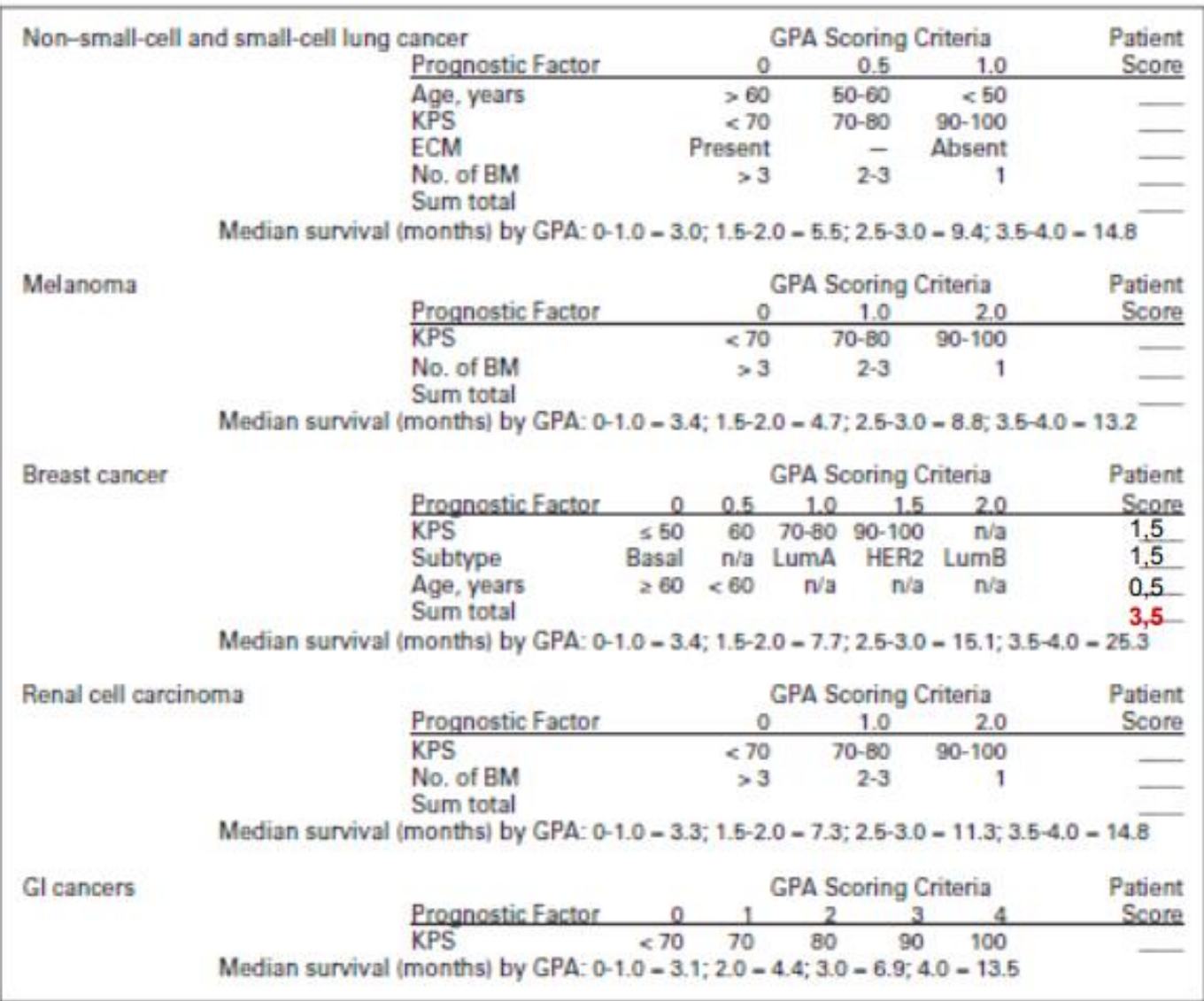




\begin{tabular}{|c|c|c|c|c|c|c|c|c|c|c|c|c|c|c|c|c|c|c|c|c|}
\hline \multirow[b]{4}{*}{ Diagnosis } & \multirow{2}{*}{\multicolumn{3}{|c|}{ Overall }} & \multicolumn{16}{|c|}{ DS-GPA Score } & \multirow{4}{*}{$\begin{array}{c}P \\
\text { [log-rank }\end{array}$} \\
\hline & & & & \multicolumn{4}{|c|}{$0-1.0$} & \multicolumn{4}{|c|}{$1.5-2.0$} & \multicolumn{4}{|c|}{$2.5-3.0$} & \multicolumn{4}{|c|}{$3.5-4.0$} & \\
\hline & \multicolumn{2}{|c|}{$\begin{array}{l}\text { Survival Time } \\
\text { (months) }\end{array}$} & \multirow{2}{*}{$\begin{array}{l}\text { No. of } \\
\text { Patients }\end{array}$} & \multicolumn{2}{|c|}{$\begin{array}{l}\text { Survival Time } \\
\text { (months) }\end{array}$} & \multicolumn{2}{|c|}{ Patients } & \multicolumn{2}{|c|}{$\begin{array}{l}\text { Survival Time } \\
\text { (months) }\end{array}$} & \multicolumn{2}{|c|}{ Patients } & \multicolumn{2}{|c|}{$\begin{array}{l}\text { Survival Time } \\
\text { (months) }\end{array}$} & \multicolumn{2}{|c|}{ Patients } & \multicolumn{2}{|c|}{$\begin{array}{c}\text { Survival Time } \\
\text { (months) }\end{array}$} & \multicolumn{2}{|c|}{$\underline{\text { Patients }}$} & \\
\hline & Median & $95 \% \mathrm{Cl}$ & & Median & $95 \% \mathrm{Cl}$ & No. & $\%$ & Median & $95 \% \mathrm{Cl}$ & No. & $\% \mathrm{~N}$ & Median & $95 \% \mathrm{Cl}$ & No. & $\% 1$ & Median & $95 \% \mathrm{Cl}$ & No. & $\%$ & \\
\hline$\overline{\mathrm{NSCLC}}$ & 7.00 & 6.53 to 7.50 & 1,833 & 3.02 & 2.63 to 3.84 & 254 & 14 & 5.49 & 4.83 to 6.40 & 705 & 38 & 9.43 & 8.38 to 10.80 & 713 & 40 & 14.78 & 11.80 to 18.80 & 161 & 9 & $<.001$ \\
\hline SCLC & 4.90 & 4.30 to 6.20 & 281 & 2.79 & 1.83 to 3.12 & 65 & 23 & 4.90 & 4.04 to 6.51 & 119 & 42 & 7.67 & 6.27 to 9.13 & 84 & 30 & 17.05 & 4.70 to 27.43 & 13 & 5 & $<.001$ \\
\hline Melanoma & 6.74 & 5.90 to 7.56 & 481 & 3.38 & 2.53 to 4.27 & 84 & 17 & 4.70 & 4.07 to 5.39 & 150 & 31 & 8.77 & 6.74 to 10.77 & 135 & 28 & 13.23 & 9.13 to 15.64 & 112 & 23 & $<.001$ \\
\hline RCC & 9.63 & 7.66 to 10.91 & 286 & 3.27 & 2.04 to 5.10 & 43 & 15 & 7.29 & 3.73 to 10.91 & 76 & 27 & 11.27 & 8.80 to 14.80 & 104 & 36 & 14.77 & 9.73 to 19.79 & 63 & 22 & $<.001$ \\
\hline Breast cancer & 13.80 & 11.53 to 15.87 & 400 & 3.35 & 3.13 to 3.78 & 23 & 6 & 7.70 & 5.62 to 8.74 & 104 & 26 & $15.07 \mid$ & 12.94 to 15.87 & 140 & 35 & 25.30 & 23.10 to 26.51 & 133 & 33 & $<.001$ \\
\hline Gl cancer & 5.36 & 4.30 to 6.30 & 209 & 3.13 & 2.37 to 4.57 & 76 & 36 & 4.40 & 3.37 to 6.53 & 65 & 31 & 6.87 & 4.86 to 11.63 & 50 & 24 & 13.54 & 9.76 to 27.12 & 18 & 9 & $<.001$ \\
\hline Other & 6.37 & 5.22 to 7.49 & 450 & - & - & - & - & - & - & - & - & $=$ & - & - & - & - & - & - & - & - \\
\hline Total & 7.16 & 6.83 to 7.52 & 3.940 & 3.10 & 2.83 to 3.45 & 545 & 16 & 5.40 & 4.90 to 5.89 & 1,219 & 35 & 9.63 & 8.74 to 10.58 & 1,226 & 35 & 16.73 & 14.65 to 18.80 & 500 & 14 & $<.001$ \\
\hline
\end{tabular}

Sperduto PW, J Clin Oncol 2012

Istologia, Volume e Numero Metastasi

Preferibile istologia mammella o polmone, diametro delle metastasi $<3$ $\mathrm{cm}$ con volume cumulativo max $15 \mathrm{ml}$, in numero non superiore a 4 . Da escludere il tronco encefalico l'istologia microcitoma. Prendere in considerazione anche le metastasi cerebrali asportate fino a $4-5 \mathrm{~cm}$ di diametro per SRT frazionata del letto operatorio

Dose

\section{Se dose singola}

$<2 \mathrm{~cm} 20-24 \mathrm{~Gy}$

$2-3 \mathrm{~cm} 18 \mathrm{~Gy}$

3-4 cm 15Gy

$>4 \mathrm{~cm}$ considerare la chirurgia.

\section{Se dose frazionata:}

$24 \mathrm{~Gy} / 3 \mathrm{fr}$

$30 \mathrm{~Gy} / 5 \mathrm{fr}$

$36 \mathrm{~Gy} / 6 \mathrm{fr}$

$25 \mathrm{~Gy} / 5 \mathrm{fr}$

$27 \mathrm{~Gy} / 3 \mathrm{fr}$ 


\section{Centratura}

TC con sezioni 1-2 mm spessore.

RM con mdc da coregistrare, possibilmente sezioni $1 \mathrm{~mm}$ spessore per intercettare micrometastasi.

Per le metastasi asportate necessaria RM pre e post (20-30 gg) intervento.

\section{Immobilizzazione}

Maschera 3 ganci rinforzata, sistema testa-collo IMRT, appoggiatesta plexiglass trasparente+ cuscino termoplastico (lasciato in acqua sopra $\mathrm{i}$ $70^{\circ}$ per 2 ore). Riferimenti su maschera, su cuscino e su base.

\section{Contornamento}

Coregistrazione della TC di centratura ed RM con mezzo di contrasto con spessore $1 \mathrm{~mm}$

GTV: area di enhancement all'esame RM-TC dopo mezzo di contrasto. L'eventuale edema non deve essere compreso.

Da GTV a PTV espansione massima $2 \mathrm{~mm}-3 \mathrm{~mm}$. Per pazienti operati espansione di 2-3 $\mathrm{mm}$ dalla presa di contrasto del cavo chirurgico.

\section{CONSTRAINTS per dose singola}

Vie ottiche: $\mathrm{D} \max (0,1 \mathrm{~cm} 3) 8 \mathrm{~Gy}$,

Tronco Encefalico: D max $(0,1 \mathrm{~cm} 3) 12 \mathrm{~Gy}$

Encefalo sano: $\mathrm{V} 10-\mathrm{V} 12<10 \mathrm{~cm} 3$ (range $5-15 \mathrm{~cm} 3$ )

Coclea: D max <12-14 Gy, D media <4-6 Gy

Cristallino: D $\max (0,1 \mathrm{~cm} 3)<1,5 \mathrm{~Gy}$

Orbita: D max $(0,1 \mathrm{~cm} 3)<8$ Gy

CONSTRAINTS per dose frazionata (30 Gy/5 fr)

Vie ottiche: D max 23Gy (5 frazioni)

Tronco Encefalico: D max $31.2 \mathrm{~Gy}$ (5 frazioni)

Encefalo sano: $\vee 28<7 \mathrm{~cm} 3$

Coclea: D media < 8-17 Gy (3 sedute), <25 Gy (5 frazioni) 


\section{Esecuzione del Trattamento}

- Copertura steroidea consueta o se il paziente non fosse già trattato con steroiode prescrivere 4-8 mg Desametasone im /die da scalare al termine della procedura

- Clip box che contenga parte della teca cranica possibilmente con strutture ben identificabili (ad es. orbite).

- EXAPOD.

- Cone beam con doppio match (bone) per tutte le sedute + EPID la prima seduta soltanto.

- Il posizionamento viene considerato ok se sta entro i limiti di correzione del Linac (5 $\mathrm{mm}$ per le traslazioni e $3^{\circ}$ per le rotazioni), altrimenti si riposiziona il paziente.

\section{Tossicita"}

Modesta. La RN (radionecrosi) dipende da tecnica, dose, volume e sede irradiati, volume di tessuto sano irradiato, pregressi trattamenti RT. Vi è una chiara

correlazione fra $\mathrm{RN}$ e $\mathrm{V} 10(>4,3 \mathrm{~cm} 3)$ e $\mathrm{V} 12(>3,3 \mathrm{~cm} 3)$. Incidenza cumulativa entro il $3 \%$. Considerare utilità del Bevacizumab nella terapia della RN.

\section{Follow-Up}

RM encefalo con mezzo di contrasto a 30-40 gg dalla SRT per verificare lo stato edemigeno, la possibile radionecrosi e la precoce risposta locale.

Secondo follow up dopo ulteriori 3 mesi sempre con RM con mezzo di contrasto e così via fino ad 1 aa quando è possibile allungare i tempi di latenza dei controlli strumentali.

Di particolare importanza la valutazione delle sequenze in diffusione, ADC (Apparent Diffusion Coefficient), delle sequenze DCE (Dynamic Contrast Enhanced) e della spettroscopia RM. 


\section{POLMONE}

\section{Criteri di Inclusione:}

- Carcinoma non a piccole cellule del polmone (NSCLC) T1-T2a NOMO con conferma bioptica o prova radiologica (criterio evolutivo) e metabolica di malignità.

- Malattia non operabile per motivi internistici o funzionali o rifiuto della chirurgia.

- Localizzazioni secondarie polmonari (diametro max $<5 \mathrm{~cm}$ ) in malattia oligometastatica, oligo-progressiva/ricorrente od oligoresponsiva a tp medica.

- Performance Status ECOG 0-2.

- Funzionalità respiratoria accettabile (suggeriti FEV $1 \geq 40 \%$ dell'atteso e DLCO $\geq 40 \%$ dell'atteso).

- Nessuna infezione polmonare, pericardica o sistemica.

Work-Up:

- TC encefalo, torace e addome superiore con mdc più recente di 8 settimane (misurazione lesione in TC).

- 18F-FDG TC-PET recente (meno di 8 settimane).

- In caso di versamento pleurico eventuale toracentesi con esame chimico-fisico e citologico.

- Prove di funzionalità respiratoria (PFR) più recenti di 8 settimane.

- Valutazione multidisciplinare.

Sistema di Immobilizzazione e Set-Up:

Abbiamo creato uno schema allegato in cartella che riassume tutte le tipologie di posizionamento possibile secondo in base agli accessori disponibili per l'esecuzione del trattamento stereotassico a livello polmonare.

\section{Definizione Volumi Bersaglio e Organi a Rischio:}

La malattia macroscopica (GTV) viene definita sulla TC di planning con finestra per parenchima polmonare e sulle altre 2 TAC co-registrate in fase di INSPIRIO ed ESPIRIO. 
Per la definizione del GTV è possibile avvalersi della fusione con immagini TC diagnostiche con mdc e/o immagini TC-PET. Non è prevista l'inclusione di ulteriori volumi per possibile coinvolgimento da parte di malattia microscopica (il CTV coincide con GTV). La creazione del ITV paziente-specifico avviene dalla somma dei 3 GTV (respiro libero-INSPESP). II PTV si ottiene aggiungendo al GTV un margine non isotropico, di $10 \mathrm{~mm}$ in direzione cranio-caudale e di $5 \mathrm{~mm}$ nelle altre direzioni.

Sono organi a rischio (OAR): midollo spinale, polmone destro, polmone sinistro, esofago, cuore, fegato, parete toracica, trachea e bronchi principali, grossi vasi mediastinici, plesso brachiale.

Tecnica di Trattamento:

3DCRT O IMRT con tecnica volumetrica. Fotoni X da $6 \mathrm{MV}$.

Prescrizione della Dose:

Prescrizione della dose sull'isodose dell' $80 \%$

Hot spot (>105\% della dose di prescrizione) all'interno del PTV.

Tabella 2: dose/frazione in base a localizzazione e stadio

\begin{tabular}{|c|c|}
\hline LOCALIZZAZIONE & DOSE TOTALE/NºRAZIONI \\
\hline $\begin{array}{l}\text { Lesioni Tla-Tlb (periferiche e }>1 \mathrm{~cm} \text { dalla parete } \\
\text { toracica) }\end{array}$ & 54 Gy/3 fr. (NCCN: 45-60/3fr) \\
\hline $\begin{array}{l}\text { da parete toracica } \\
\text { desioni Tla- T2a (centrali o periferiche }<4-5 \mathrm{~cm}\end{array}$ & $\mathrm{NCCl}$ \\
\hline $\begin{array}{l}\text { Lesioni centrali (entro } 2 \mathrm{~cm} \text { dall'albero bronchiale } 0 \\
\text { entro } 1 \mathrm{~cm} \text { dai grossi vasi mediastinici) }\end{array}$ & $\begin{array}{l}60 \text { Gy/8 fr.(NCCN: 60-70Gy/ } \\
\text { 8-10fr. }\end{array}$ \\
\hline Lesioni T2a o lesioni Tl adese alla parete torc & $55 \mathrm{G}$ \\
\hline
\end{tabular}


Figura 2: schema di localizzazione delle lesioni

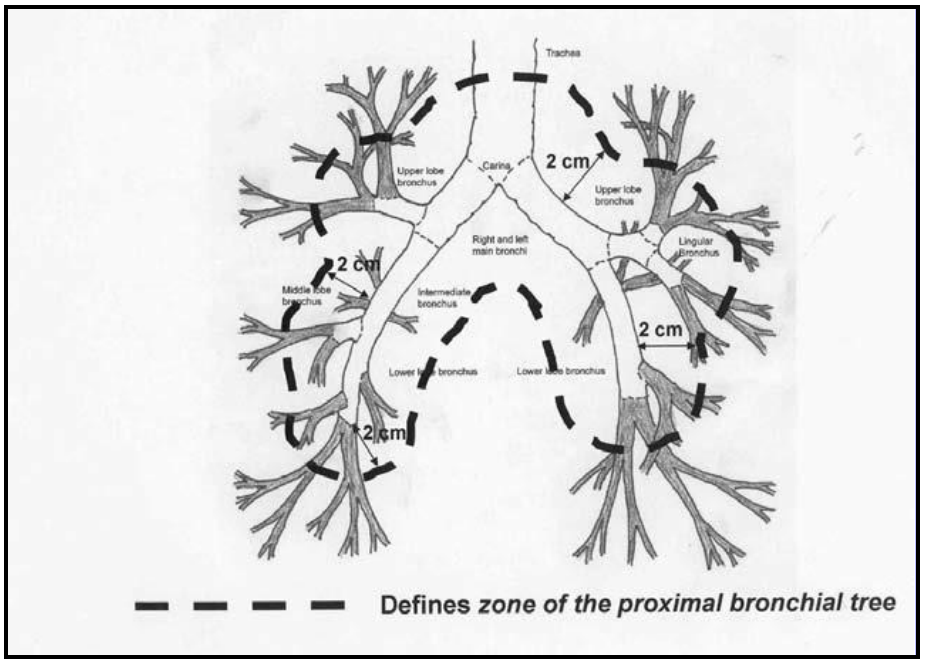

Limiti di Dose ai Tessuti Sani (ref. Dose tolerance Limits and dose histogram evaluation for SBRT. Grimm J et al. Journal of Applied Clincal Medical Physics. Vol 12, num2, 2011 ).

$\mathrm{MLD}_{2}$ polmone omolaterale < $12 \mathrm{~Gy}$ 
Tabella 3: constraints di dose

\begin{tabular}{|c|c|c|c|c|c|c|c|c|}
\hline \multirow[t]{2}{*}{ Tessuti seriali } & \multirow{2}{*}{$\begin{array}{l}\text { Volume } \\
\text { max } \\
\text { oltre la } \\
\text { dose } \\
\text { soglia }\end{array}$} & \multicolumn{2}{|c|}{ Singola frazione } & \multicolumn{2}{|l|}{3 frazioni } & \multicolumn{2}{|l|}{ 5-8 frazioni } & \multirow[t]{2}{*}{ Tossicità ( $\geq \mathbf{G} 3$ ) } \\
\hline & & $\begin{array}{l}\text { Dose } \\
\text { soglia } \\
\text { (Gy } \\
{[\text { Gy/fr.]) }}\end{array}$ & $\begin{array}{l}\text { Dose } \\
\max \\
(\mathrm{Gy} \\
[\mathrm{Gy} / \mathrm{fr} .])\end{array}$ & $\begin{array}{l}\text { Dose } \\
\text { soglia } \\
\text { (Gy } \\
\text { [Gy/fr.]) }\end{array}$ & $\begin{array}{l}\text { Dose max } \\
\text { (Gy } \\
[G y / f r .])\end{array}$ & $\begin{array}{l}\text { Dose soglia } \\
\text { (Gy [Gy/fr.]) }\end{array}$ & $\begin{array}{l}\text { Dose } \\
\max \\
\text { (Gy } \\
[\mathrm{Gy} / \mathrm{fr} .])\end{array}$ & \\
\hline $\begin{array}{l}\text { Midollo } \\
\text { spinale } \\
\text { Midollo } \\
\text { allungato } \\
\end{array}$ & $\begin{array}{l}<0.35 \mathrm{cc} \\
<1.2 \mathrm{cc}\end{array}$ & $\begin{array}{l}10 \\
7\end{array}$ & 14 & $\begin{array}{l}18(6) \\
12.3(4.1)\end{array}$ & $21.9(7.3)$ & $\begin{array}{l}23(4.6) \\
14.5(2.9)\end{array}$ & $30(6)$ & Mielite \\
\hline $\begin{array}{l}\text { Midollo } \\
\text { spinale } \\
\text { (sottovolume } \\
\text { sec. Ryu) }\end{array}$ & $<10 \%$ & 10 & 14 & $18(6)$ & $21.9(7.3)$ & $23(4.6)$ & $30(6)$ & Mielite \\
\hline Esofago & $<5 \mathrm{CC}$ & 11.9 & 15.4 & $17.7(5.9)$ & $25.2(8.4)$ & $19.5(3.9)$ & $35(7)$ & Stenosi/ fistola \\
\hline $\begin{array}{l}\text { Plesso } \\
\text { brachiale }\end{array}$ & $<3 \mathrm{CC}$ & 14 & 17.5 & $20.4(6.8)$ & $24(8)$ & $27(5.4)$ & $30.5(6.1)$ & Neuropatia \\
\hline $\begin{array}{l}\text { Cuore/perica } \\
\text { rdio }\end{array}$ & $<15 \mathrm{cC}$ & 16 & 22 & $24(8)$ & $30(10)$ & $32(6.4)$ & $38(7.6)$ & Pericardite \\
\hline Grandi vasi & $<10 \mathrm{cc}$ & 31 & 37 & $39(13)$ & $45(15)$ & $47(9.4)$ & $53(10.6)$ & Aneurisma \\
\hline $\begin{array}{l}\text { Trachea/bron } \\
\text { chi principali }\end{array}$ & $<4 \mathrm{cc}$ & 10.5 & 20.2 & $15(5)$ & $30(10)$ & $16.5(3.3)$ & $40(8)$ & Stenosi/fistola \\
\hline $\begin{array}{l}\text { Piccole vie } \\
\text { aeree }\end{array}$ & $<0.5 \mathrm{cc}$ & 12.4 & 13.3 & $18.9(6.3)$ & $23.1(7.7)$ & $21(4.2)$ & $33(6.6)$ & Stenosi/atelettasia \\
\hline Coste & $\begin{array}{l}<1 \mathrm{cC} \\
<30 \mathrm{cc}\end{array}$ & 22 & 30 & $\begin{array}{l}28.8(9.6) \\
30(10)\end{array}$ & $\begin{array}{l}36.9(12.3) \\
\text { D0,5 CC } \\
(\text { costa) } \\
<33 G y\end{array}$ & $35(7)$ & $43(8.6)$ & Dolore/frattura \\
\hline Cute & $<10 \mathrm{cc}$ & 23 & 26 & $30(10)$ & $33(11)$ & $36.5(7.3)$ & $39.5(7.9)$ & Ulcerazione \\
\hline $\begin{array}{l}\text { Tessuti } \\
\text { paralleli }\end{array}$ & & & & & & & & \\
\hline $\begin{array}{l}\text { Polmone } \\
\text { (ds/sn) }\end{array}$ & $\begin{array}{l}1500 \mathrm{cc} \\
1000 \mathrm{cc}\end{array}$ & $\begin{array}{l}7 \\
7.4\end{array}$ & & $\begin{array}{l}11.6(2.9) \\
12.4(3.1)\end{array}$ & & $\begin{array}{l}12.5(2.5) \\
13.5(2.7) \\
\text { V5 bilat } \\
<37 \% \\
\text { V5 } \\
\text { controlat } \\
26 \%\end{array}$ & & $\begin{array}{l}\text { Funzione polmonare } \\
\text { Polmonite }\end{array}$ \\
\hline Fegato & $700 \mathrm{cc}$ & 9.1 & & $19.2(4.8)$ & & $21(4.2)$ & & Funzione epatica \\
\hline
\end{tabular}




\section{Somministrazione:}

Posizionamento del paziente nel presidio di immobilizzazione con compressore addominale (se utilizzato in TC) e localizzatore Hexapod.

ConeBeam CT quotidiana con correzione degli spostamenti e degli angoli di rotazione on-line

Nella procedura CBCT si farà riferimento ad una Clip Box che vada a comprendere il PTV, con protocollo di fusione Grey Value (nella filosofia del trattamento stereotassico frame-less in cui il tumore stesso funge da fiduciale). E' necessario il controllo del match da parte del medico.

Follow-Up:

Definizione della risposta secondo i criteri RECIST.

Valutazione della tossicità clinica acuta e cronica secondo scala RTOG.

Prima visita di follow-up con visita clinica e TC encefalo, torace, addome superiore con mdc a 45-60 giorni dal termine.

Visite successive con TC encefalo, torace e addome superiore con mdc ogni 3 mesi per 1 anno e quindi ogni 6 mesi fino a 5 anni.

Non raccomandata routinariamente l'esecuzione di TC-PET nel follow-up e comunque successiva ad almeno 3 mesi dal trattamento SBRT.

\section{METASTASI LINFONODALI DI TUMORI SOLIDI}

\section{Criteri di Inclusione}

- conferma istologica del tumore primario;

- pazienti olimetastatici/oligoricorrenti/oligoprogressivi con $\leq 5$ lesioni (con dimensioni $\max \leq 3 \mathrm{~cm}$ ), $0 \leq 3$ lesioni (con dimensioni max $\leq 4 \mathrm{~cm}$ );

- tumore primitivo controllato;

- disponibilità di imaging (PET con radiofarmaco appropriato) \pm RM/TC recente (da valutare in base all'istologia);

- Buon PS

$-$

\section{Criteri di Esclusione}

- pazienti con oltre 5 lesioni linfonodali;

- prognosi < 3 mesi;

- tumore primitivo non controllato.

Imaging Pre-R†

Dovrebbero essere eseguiti TC ( \pm RM) con mdc e PET (eventualmente di centratura) con radiofarmaco idoneo. 


\section{TC di Simulazione}

Al fine di consentire un accurato contouring del target volume e degli organi a rischio deve essere eseguita TC di centratura in posizione di trattamento con slices 1,25 che potrà essere coregistrata con le immagini TC e/o PET e/o RM disponibili.

\section{Posizionamento}

A seconda della sede cervicale, toracica, addominale o pelvica viene valutato paziente per paziente il posizionamento migliore, anche il relazione ad eventuale trattamento eseguito in precedenza in aree limitofre.

\section{Contouring}

La delineazione del target volume verrà eseguita come segue:

- il GTV verrà delineato, come malattia visibile, sulle immagini TC di simulazione eventualmente in fusione con le immagini diagnostiche;

- non verrà aggiunto un margine al GTV al fine di ottenere il CTV (CTV = GTV);

- per ottenere il PTV verrà aggiunto un margine isotropico di 3-5 mm (in funzione della sede linfonodale e degli OaR);

- delineare tutti gli altri OaR inclusi nella regione corporea.

Dosi

8 Gy X 3 Frazioni giornaliere = 24 Gy (EQD2 circa 72 Gy con alfa/beta= 1; EQD2 circa 53 Gy con alfa/beta $=3$ )

7 Gy x4 frazioni = 28 Gy (EQD2 circa 75 Gy, con alfa/beta 1 e 56 Gy con alfa/beta 3)

7 Gy $x 5$ frazioni $=35$ Gy (EQD2 circa 93 Gy con alfa/beta 1 eEQD2 circa 70 con alfa/beta 3 )

6 Gy $\times 5$ frazioni $=30$ Gy (EQD2 circa 70 Gy con alfa/beta 1 eEQD2 circa 54 con alfa/beta 3 )

\section{Dose Constraints}

In base alla sede di trattamento i limiti di dose devono tenere conto degli organi a rischio presenti in tale sede e dell'eventuale pregressa radioterapia in sedi limitrofe. 


\section{Tabella 4: dose constraints}

Thoracic dose constraints

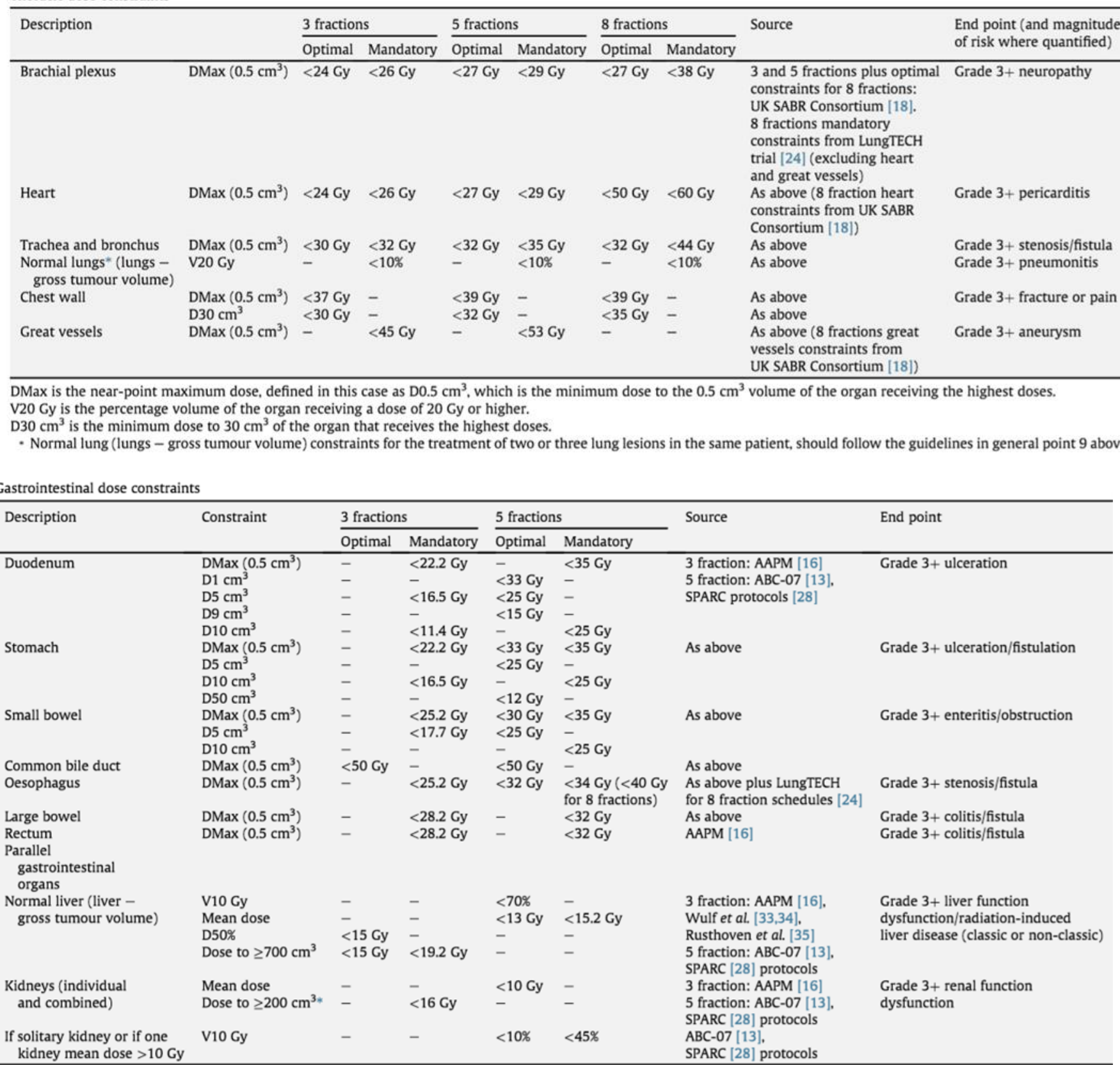

DMax is the near-point maximum dose, defined in this case as D0.5 $\mathrm{cm}^{3}$, which is the minimum dose to the $0.5 \mathrm{~cm}^{3}$ volume of the organ receiving the highest doses,

$\mathrm{D} 1 \mathrm{~cm}^{3}, \mathrm{D} 5 \mathrm{~cm}^{3}, \mathrm{D} 9 \mathrm{~cm}^{3}, \mathrm{D} 10 \mathrm{~cm}^{3}$ and D50 cm $\mathrm{cm}^{3}$ are the minimum doses to the specified volume of the organ $\left(1 \mathrm{~cm}^{3}, 5 \mathrm{~cm}^{3}\right.$, etc.) that receive the highest doses.

$\mathrm{V} 10 \mathrm{~Gy}$ is the percentage volume of the organ receiving a dose of $10 \mathrm{~Gy}$ or higher.

Dose to $\geq 700 \mathrm{~cm}^{3}$ and $\geq 200 \mathrm{~cm}^{3}$ is the maximum dose to the specified volume of the organ $\left(700 \mathrm{~cm}^{3}, 200 \mathrm{~cm}^{3}\right)$ that receives the lowest doses.

- If total kidney volume $<200 \mathrm{~cm}^{3}$, or treating renal or adrenal lesions, then total dose to contralateral kidney should be $<16 \mathrm{~Gy}$ and aim to minimise spillage into ipsilateral kidney if possible. 
Pelvic dose constraints (for non-prostate primary irradiation)

\begin{tabular}{|c|c|c|c|c|c|c|c|}
\hline \multirow[t]{2}{*}{ Description } & \multirow[t]{2}{*}{ Constraint } & \multicolumn{2}{|l|}{3 fractions } & \multicolumn{2}{|l|}{5 fractions } & \multirow[t]{2}{*}{ Source } & \multirow[t]{2}{*}{ End point } \\
\hline & & Optimal (Gy) & Mandatory (Gy) & Optimal (Gy) & Mandatory (Gy) & & \\
\hline Bladder & $\begin{array}{l}D 15 \mathrm{~cm}^{3} \\
\operatorname{DMax}\left(0.5 \mathrm{~cm}^{3}\right)\end{array}$ & $\bar{z}$ & $\begin{array}{l}<16.8 \\
<28.2\end{array}$ & $\overline{-}$ & $\begin{array}{l}<18.3 \\
<38\end{array}$ & MAPM [16] & Grade $3+$ cystitis/fistula \\
\hline
\end{tabular}

DMax is the near-point maximum dose, defined in this case as DOS $\mathrm{cm}^{3}$. which is the minimum dose to the $0.5 \mathrm{~cm}^{3}$ volume of the organ

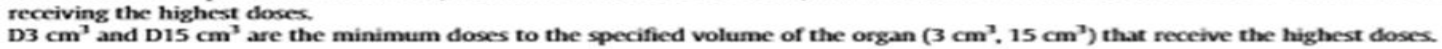

\section{Esecuzione Del Trattamento}

Posizionamento del paziente con il presidio di immobilizzazione scelto in TC di simulazione e posizionamento del localizzatore Exapod.

CLIP BOX che comprenda il target volume e le strutture anatomiche adiacenti utili per il matching.

CBCT quotidiana e matching delle immagini utilizzando il protocollo di fusione scelto dal medico (grey value, bone o altro), in base alla sede linfonodale.

Correzione quotidiana on-line degli spostamenti nelle diverse direzioni dello spazio.

Follow-Up

Il follow up sarà impostato in base al tumore primitivo. 


\section{BIBLIOGRAFIA}

1. AIRO. La Radioterapia stereotassica nel trattamento della malattia oligometastatica. Luglio 2019.

2. Amsbaugh MJ, Boling $\mathrm{W}$, Woo $\mathrm{S}$. Tumor bed radiosurgery: an emerging treatment for brain metastases. J Neurooncol. 2015 Jun;123(2):197-203. doi: 10.1007/s11060-015-1789-y. Epub 2015 Apr 25.

3. Aoyama H, Shirato H, Tago M, Nakagawa K, Toyoda T, Hatano K, Kenjyo $M$, Oya $N$, Hirota $S$, Shioura $H$, Kunieda $E$, Inomata $T$, Hayakawa K, Katoh N, Kobashi $G$. Stereotactic radiosurgery plus whole-brain radiation therapy vs stereotactic radiosurgery alone for treatment of brain metastases: a randomized controlled trial. JAMA. 2006 Jun 7;295(21):2483-91.

4. Atalar B, Modlin LA, Choi CY, Adler JR, Gibbs IC, Chang SD, Harsh GR 4th, Li G, Nagpal S, Hanlon A, Soltys SG. Risk of leptomeningeal disease in patients treated with stereotactic radiosurgery targeting the postoperative resection cavity for brain metastases. Int J Radiat Oncol Biol Phys. 2013 Nov 15;87(4):713-8. doi: 10.1016/j.jijrobp.2013.07.034. Epub 2013 Sep 18.

5. Auchter RM, Lamond JP, Alexander E, Buatti JM, Chappell R, Friedman WA, Kinsella TJ, Levin AB, Noyes WR, Schultz CJ, Loeffler JS, Mehta MP. A multiinstitutional outcome and prognostic factor analysis of radiosurgery for resectable single brain metastasis. Int J Radiat Oncol Biol Phys. 1996 Apr 1;35(1):27-35.

6. Bhattacharya IS, Woolf DK, Hughes RJ, Shah N, Harrison M, Ostler PJ, Hoskin PJ. Stereotactic body radiotherapy (SBRT) in the management of extracranial oligometastatic (OM) disease. $\mathrm{Br} \mathrm{J}$ Radiol. 2015 Apr;88(1048):20140712. doi: 10.1259/bjr.20140712. Epub 2015 Feb 13. 
7. Benedict SH, Yenice KM, Followill D, Galvin JM, Hinson W, Kavanagh B, Keall P, Lovelock M, Meeks S, Papiez L, Purdie T, Sadagopan R, Schell MC, Salter B, Schlesinger DJ, Shiu AS, Solberg T, Song DY, Stieber $V$, Timmerman $R$, Tomé WA, Verellen D, Wang L, Yin FF. Stereotactic body radiation therapy: the report of AAPM Task Group 101. Med Phys. 2010 Aug;37(8):4078-101.

8. Chen GT, Kung JH, Beaudette KP. Artifacts in computed tomography scanning of moving objects. Semin Radiat Oncol. 2004 Jan;14(1):19-26.

9. Chung $\mathbf{H}$, Jin $\mathrm{H}$, Palta J, SUh TS, Kim S. Dose variations with varying calculation grid size in head and neck IMRT. Phys Med Biol. 2006 Oct 7:51 (19):4841-56. Epub 2006 Sep 14.

10. Cox BW, Spratt DE, Lovelock M, Bilsky MH, Lis E, Ryu S, Sheehan J, Gerszten PC, Chang E, Gibbs I, Soltys S, Sahgal A, Deasy J, Flickinger J, Quader M, Mindea S, Yamada Y. International Spine Radiosurgery Consortium consensus guidelines for target volume definition in spinal stereotactic radiosurgery. Int $\mathrm{J}$ Radiat Oncol Biol Phys. 2012 Aug 1;83(5):e597-605. doi: 10.1016/j.jirobp.2012.03.009. Epub 2012 May 19.

11. Eaton BR, LaRiviere MJ, Kim S, Prabhu RS, Patel K, Kandula S, Oyesiku N, Olson J, Curran W, Shu HK, Crocker I. Hypofractionated radiosurgery has a befter safety profile than single fraction radiosurgery for large resected brain metastases. J Neurooncol. 2015 May;123(1):103-11. doi: 10.1007/s 11060-015-1767-4. Epub 2015 Apr 11.

12. Gambhir SS, Czernin J, Schwimmer J, Silverman DH, Coleman RE, Phelps ME. A tabulated summary of the FDG PEt literature. J NuCl Med. 2001 May;42(5 Suppl):1S-93S.

13. Garcia-Barros M, Paris F, Cordon-Cardo C, Lyden D, Rafii S, Haimovitz-Friedman A, Fuks Z, Kolesnick R. Tumor response to 
radiotherapy regulated by endothelial cell apoptosis. Science. 2003 May 16;300(5622):1 155-9.

14. Greco C, Zelefsky MJ, Lovelock M, Fuks Z, Hunt M, Rosenzweig K, Zatcky J, Kim B, Yamada Y. Predictors of local control after singledose stereotactic image-guided intensity-modulated radiotherapy for extracranial metastases. Int J Radiat Oncol Biol Phys. 2011 Mar 15;79(4):1 151-7. doi: 10.1016/j.jijrobp.2009.12.038. Epub 2010 May 25.

15. Grimm J, LaCouture T, Croce R, Yeo I, Zhu Y, Xue J. Dose tolerance limits and dose volume histogram evaluation for stereotactic body radiotherapy. J Appl Clin Med Phys. 2011 Feb 8;12(2):3368.

16. Guckenberger $M$, Aerts JG, Van Schil P, Weder W. The American Society of Clinical Oncology-endorsed American Society for Radiation Oncology Evidence-Based Guideline of stereotactic body radiotherapy for early-stage non-small cell lung cancer: An expert opinion. J Thorac Cardiovasc Surg. 2019 Jan;157(1):358-361. doi: 10.1016/j.jtcvs.2018.09.107. Epub 2018 Oct 12.

17. Guckenberger $\quad \boldsymbol{M}$, Lievens $\quad \mathrm{Y}$, Bouma $\quad \mathrm{A}$ 3, Collette $\quad \mathbf{L}$, Dekker A, deSouza NM, Dingemans AC, Fournier B, Hurkmans C, Lecouvet FE, Meattini I, Romero AM, Ricardi U, Russell NS, Schanne DH, Scorsetti M, Tombal B, Verellen D, Verfaillie C, Ost P. Characterisation and classification of oligometastatic disease: a European Society for Radiotherapy and Oncology and European Organisation for Research and Treatment of Cancer consensus recommendation. Lancet Oncol. 2020 Jan;21(1):e18-e28. doi: 10.1016/S1470-2045(19)30718-1.

18. Günther P, Schenk JP, Wunsch R, Tröger J, Waag KL. Abdominal tumours in children: 3-D visualisation and surgical planning. Eur $J$ Pediatr Surg. 2004 Oct; 14(5):316-21. 
19. Gupta NC, Graeber GM, Tamim WJ, Rogers JS, Irisari L, Bishop HA. Clinical utility of PET-FDG imaging in differentiation of benign from malignant adrenal masses in lung cancer. Clin Lung Cancer. 2001 Aug:3(1):59-64.

20. Hanna GG, Murray L, Patel R, Jain S, Aitken KL, Franks KN, van As N, Tree A, Hatfield P, Harrow S, McDonald F, Ahmed M, Saran FH, Webster GJ, Khoo V, Landau D, Eaton DJ, Hawkins MA. UK Consensus on Normal Tissue Dose Constraints for Stereotactic Radiotherapy. Clin Oncol (R Coll Radiol). 2018 Jan;30(1):5-14. doi: 10.1016/j.clon.2017.09.007. Epub 2017 Oct 13.

21. Hellman S, Weichselbaum RR. Oligometastases. J Clin Oncol. 1995 Jan;13(1):8-10.

22. Hsieh J, Elson P, Otvos B, Rose J, Loftus C, Rahmathulla G, Angelov $L$, Barnett GH, Weil RJ, Vogelbaum MA. Tumor progression in patients receiving adjuvant whole-brain radiotherapy vs localized radiotherapy after surgical resection of brain metastases. Neurosurgery. 2015 Apr;76(4):411-20. doi: $10.1227 /$ NEU.0000000000000626.

23. Jereczek-Fossa BA, Beltramo G, Fariselli L, Fodor C, Santoro L, Vavassori A, Zerini D, Gherardi F, Ascione C, Bossi-Zanetti I, Mauro R, Bregantin A, Bianchi LC, De Cobelli O, Orecchia R. Robotic imageguided stereotactic radiotherapy, for isolated recurrent primary, lymph node or metastatic prostate cancer. Int J Radiat Oncol Biol Phys. 2012 Feb 1;82(2):889-97. doi: 10.1016/j.jjrobp.2010.11.031. Epub 2011 Jan 27.

24. Jereczek-Fossa BA, Bossi-Zanetti I, Mauro R, Beltramo G, Fariselli L, Bianchi LC, Fodor C, Fossati P, Baroni G, Orecchia R. CyberKnife robotic image-guided stereotactic radiotherapy for oligometastic cancer: A prospective evaluation of 95 patients/118 lesions. 
Strahlenther Onkol. 2013 Jun;189(6):448-55. doi: 10.1007/s00066-0130345-y. Epub 2013 Apr 21.

25. Jereczek-Fossa BA, Piperno G, Ronchi S, Catalano G, Fodor C, Cambria R, Fossati Ing P, Gherardi F, Alterio D, Zerini D, Garibaldi C, Baroni G, De Cobelli O, Orecchia R. Linac-based stereotactic body radiotherapy for oligometastatic patients with single abdominal lymph node recurrent cancer. Am J Clin Oncol. 2014 Jun;37(3):22733. doi: 10.1097/COC.0b013e3182610878

26. Kocher M, Soffietti R, Abacioglu U, Villà S, Fauchon F, Baumert BG, Fariselli L, Tzuk-Shina T, Kortmann RD, Carrie C, Ben Hassel M, Kouri M, Valeinis E, van den Berge D, Collette S, Collette L, Mueller RP. Adjuvant whole-brain radiotherapy versus observation after radiosurgery or surgical resection of one to three cerebral metastases: results of the EORTC 22952-26001 study. J Clin Oncol. 2011 Jan 10;29(2):134-41. doi: 10.1200/JCO.2010.30.1655. Epub 2010 Nov 1.

27. Kollar L, Rengan R. Stereotactic body radiotherapy. Semin Oncol. 2014 Dec;41(6):776-89. doi: 10.1053/j.seminoncol.2014.09.022. Epub 2014 Oct 7.

28. Komaki R, Mountain CF, Holbert JM, Garden AS, Shallenberger R, Cox JD, Maor MH, Guinee VF, Samuels B. Superior sulcus tumors: treatment selection and results for 85 patients without metastasis (Mo) at presentation. Int J Radiat Oncol Biol Phys. 1990 Jul;19 (1):31-6

29. Lardinois D, Weder W, Hany TF, Kamel EM, Korom S, Seifert B, von Schulthess GK, Steinert HC. Staging of non-small-cell lung cancer with integrated positron-emission tomography and computed tomography. N Engl J Med. 2003 Jun 19;348(25):2500-7.

30. Mahadevan A, Bucholz R, Gaya AM, Kresl JJ, Mantz C, Minnich DJ, Muacevic A, Medbery C 3rd, Yang J, Caglar HB, Davis JN. Best of 
the Radiosurgery Society ${ }^{\circledR}$ Scientific Meeting 2014: stereotactic radiosurgery/stereotactic body radiotherapy treatment of extracranial and intracranial lesions. Future Oncol. 2014 Dec;10(15):2307-10. doi: 10.2217/fon.14.168.

31. Matsushita $\mathbf{H}$, Jingu $K$, Umezawa $R$, Yamamoto $T$, Ishikawa $Y$, Takahashi N, Katagiri Y, Kadoya N. Stereotactic Radiotherapy for Oligometastases in Lymph Nodes-A Review. Technol Cancer Res Treat. 2018 Jan 1;17:1533033818803597. doi: $10.1177 / 1533033818803597$.

32. McPherson CM, Suki D, Feiz-Erfan I, Mahajan A, Chang E, Sawaya R, Lang FF. Adjuvant whole-brain radiation therapy after surgical resection of single brain metastases. Neuro Oncol. 2010 Jul;12(7):71 1-9. doi: 10.1093/neuonc/noq005. Epub 2010 Feb 14

33. Milano MT, Katz AW, Muhs AG, Philip A, Buchholz DJ, Schell MC, Okunieff $P$. A prospective pilot study of curative-intent stereotactic body radiation therapy in patients with $\mathbf{5}$ or fewer oligometastatic lesions. Cancer. 2008 Feb 1;112(3):650-8.

34. Minniti G, Esposito V, Clarke E, Scaringi C, Lanzetta G, Salvati M, Raco A, Bozzao A, Maurizi Enrici R. Multidose stereotactic radiosurgery $(9 \mathrm{~Gy} \times 3$ ) of the postoperative resection cavity for treatment of large brain metastases. Int J Radiat Oncol Biol Phys. 2013 Jul 15;86(4):623-9. doi: 10.1016/j.jjrobp.2013.03.037. Epub 2013 May 15.

35. Muacevic A, Kreth FW, Horstmann GA, Schmid-Elsaesser R, Wowra $B$, Steiger HJ, Reulen HJ. Surgery and radiotherapy compared with gamma knife radiosurgery in the treatment of solitary cerebral metastases of small diameter. J Neurosurg. 1999 Jul;91 (1):35-43.

36. Muacevic A, Wowra B, Siefert A, Tonn JC, Steiger HJ, Kreth FW. Microsurgery plus whole brain irradiation versus Gamma Knife 
surgery alone for treatment of single metastases to the brain: a randomized controlled multicentre phase III trial. J Neurooncol. 2008 May;87(3):299-307. Epub 2007 Dec 22.

37. Overgaard J, Gonzalez Gonzalez D, Hulshof MC, Arcangeli G, Dahl $O$, Mella $O$, Bentzen SM. Randomised trial of hyperthermia as adjuvant to radiotherapy for recurrent or metastatic malignant melanoma. European Society for Hyperthermic Oncology. Lancet. 1995 Mar 4;345(8949):540-3.

38. Roberge D, Parney I, Brown PD, Radiosurgery to the postoperative surgical cavity: who needs evidence? Int J Rad Onc Bio Phys 2012:83:486-493.doi: 10.1016/j.jijobp.201 1.09.032. Epub 2011 Nov 16.

39. Sahgal A, Aoyama H, Kocher M, Neupane B, Collette S, Tago M, Shaw P, Beyene J, Chang EL. Phase 3 trials of stereotactic radiosurgery with or without whole-brain radiation therapy for 1 to 4 brain metastases: individual patient data meta-analysis. Int J Radiat Oncol Biol Phys. 2015 Mar 15;91(4):710-7. doi: 10.1016/j.jirobp.2014.10.024.

40. Salama JK, Chmura SJ, Mehta N, Yenice KM, Stadler WM, Vokes EE, Haraf DJ, Hellman S, Weichselbaum RR. An initial report of a radiation dose-escalation trial in patients with one to five sites of metastatic disease. Clin Cancer Res. 2008 Aug 15;14(16):5255-9. doi: 10.1158/1078-0432.CCR-08-0358.

41. Seo YS, Kim MS, Yoo HJ, Jang W. Stereotactic body radiotherapy for oligo-recurrence within the nodal area from colorectal cancer. World J Gastroenterol. 2014 Feb 28;20(8):2005-13. doi: 10.3748/wjg.v20.i8.2005.

42. Sneed PK, Suh JH, Goetsch SJ, Sanghavi SN, Chappell R, Buatti JM, Regine WF, Weltman E, King VJ, Breneman JC, Sperduto PW, Mehta MP. A multi-institutional review of radiosurgery alone vs. radiosurgery with whole brain radiotherapy as the initial 
management of brain metastases. Int J Radiat Oncol Biol Phys. 2002 Jul 1;53(3):519-26.

43. Soffietti R, Kocher M, Abacioglu UM, Villa S, Fauchon F, Baumert BG, Fariselli L, Tzuk-Shina T, Kortmann RD, Carrie C, Ben Hassel M, Kouri M, Valeinis E, van den Berge D, Mueller RP, Tridello G, Collette L, Bottomley A. A European Organisation for Research and Treatment of Cancer phase III trial of adjuvant whole-brain radiotherapy versus observation in patients with one to three brain metastases from solid tumors after surgical resection or radiosurgery: quality-oflife results. J Clin Oncol. 2013 Jan 1:31(1):65-72. doi: 10.1200/JCO.2011.41.0639. Epub 2012 Dec 3.

44. Stieb S, Lang S, Linsenmeier C, Graydon S, Riesterer O. Safety of high-dose-rate stereotactic body radiotherapy. Radiat Oncol. 2015 Jan 23;10:27. doi: 10.1186/s13014-014-0317-0.

45. Thibault I, Chang EL, Sheehan J, Ahluwalia MS, Guckenberger M, Sohn MJ, Ryu S, Foote M, Lo SS, Muacevic A, Soltys SG, Chao S, Gerszten P, Lis E, Yu E, Bilsky M, Fisher C, Schiff D, Fehlings MG, Ma L, Chang S, Chow E, Parelukar WR, Vogelbaum MA, Sahgal A. Response assessment after stereotactic body radiotherapy for spinal metastasis: a report from the SPIne response assessment in Neuro-Oncology (SPINO) group. Lancet Oncol. 2015 Dec;16(16):e595-603. doi: 10.1016/S1470-2045(15)00166-7.

46. Timmerman RD. An overview of hypofractionation and introduction to this issue of seminars in radiation oncology. Semin Radiat Oncol. 2008 Oct;18(4):215-22. doi: 10.1016/j.semradonc.2008.04.001.

47. Timmerman RD, Bizekis CS, Pass HI, Fong Y, Dupuy DE, Dawson LA, LU D. Local surgical, ablative, and radiation treatment of metastases. CA Cancer J Clin. 2009 May-Jun;59(3):145-70. doi: 10.3322/caac.20013. Epub 2009 Apr 10. 
48. Tsao $M, X \cup W$, Sahgal A. A meta-analysis evaluating stereotactic radiosurgery, whole-brain radiotherapy, or both for patients presenting with a limited number of brain metastases. Cancer. 2012 May 1;118(9):2486-93. doi: 10.1002/cncr.26515. Epub 2011 Sep

49. Webb WR, Gatsonis C, Zerhouni EA, Heelan RT, Glazer GM, Francis $\mathbb{R}$, MCNeil BJ. CT and $M R$ imaging in staging non-small cell bronchogenic carcinoma: report of the Radiologic Diagnostic Oncology Group. Radiology. 1991 Mar; 178(3):705-13. 


\section{n. 03}

pubblicazione revisionata e approvata

\section{giugno 2020}

disponibile online www.ospedale.al.it/

working-papers-wp 